\title{
Perspective \\ Perspective on "The effect of shape on the interaction of colloidal particles"
}

\section{Onsager L (1949) Ann NY Acad Sci 51: 627}

\author{
Daan Frenkel \\ FOM Institute for Atomic and Molecular Physics, Kruislaan 407, 1098 SJ Amsterdam, The Netherlands
}

Received: 24 February 1999 / Accepted: 3 March 1999 / Published online: 21 June 1999

\begin{abstract}
Onsager's paper on the effect of shape on the interaction of colloidal particles is seminal in many ways. I shall focus on two aspects: it is (to my knowledge) the earliest classical density functional theory, and it demonstrates the possibility of ordering transitions driven by entropy
\end{abstract}

Key words: Entropic phase transition - Density functional theory - Nematic liquid crystal - Freezing

If ever the description "multifaceted" should apply to a paper, it is to Onsager's analysis of the effect of shape on the interaction of colloidal particles. The primary aim of this paper was to explain the physics behind the formation of an anisotropic (nematic) phase in a relatively dilute suspension of rigid, linear colloids. Here I shall not discuss those aspects of the paper that focus mainly on the analysis of the forces acting between charged colloidal rods (actually not just rods) in solution. Although this analysis makes the present paper one of the milestones in theoretical colloid physics, I would like to start where this part of the analysis ends namely with the conclusion that, to a good approximation, the interaction between charged rods in a not too dilute salt solution is short-ranged repulsion. This implies that, as a first approximation, it is permissible to treat such rodlike particles as slender, hard rods. Onsager then goes on to consider a limiting case: rods for which the ratio of length $(L)$ to the diameter $(D)$ tends to infinity. Using this description of rodlike colloids, Onsager then proceeds to show that in such a model, a transition from the isotropic to the nematic phase must occur above a certain density (of order $\left.\left(L^{2} D\right)^{-1}\right)$.

There are several features of Onsager's analysis that make this paper crucial for subsequent developments. The first is the way in which Onsager derives an expression for the free energy of a system of thin hard rods. What Onsager does is nothing less than write down the free energy of the system as a functional of the single-particle distribution function - deriving such an expression is the central aim of all subsequent densityfunctional theories, be they classical or quantummechanical (see e.g. Ref. [1]). Unfortunately, we usually do not know the true density functional, and we are forced to make approximations. Not so in Onsager's case: the density functional that Onsager writes down for infinitely thin hard rods is almost certainly exact. Personally, I would say that it is exact - it relies on the fact that all virial coefficients higher than the second are negligible: Onsager showed that this is plausible, and subsequent numerical work [2] supports this claim. This makes the Onsager density functional one of the very few that is both nontrivial and exact. Although the density functional that Onsager derived is exact, the freeenergy minimization cannot be carried out analytically. Onsager found an approximate solution, using an ingenious ansatz for the single-particle distribution function; however, numerically, the minimization can be carried out to any desired accuracy. In this sense, we now know the "exact" density dependence of the free energy of thin hard rods. The Onsager approach has proven extremely fruitful. It has been applied to a variety of model systems, including semiflexible particles [3, 4]. However, here I will not review the subsequent work, but rather discuss another aspect of Onsager's paper.

The transition from the isotropic to the nematic phase involves a partial ordering of the molecular orientations. It is common practice to consider entropy as a measure for the "disorder" in a system. A naive observer would therefore conclude that the isotropic phase must always have a higher entropy than the nematic phase at the same density and energy. In this picture, ordering can only occur due to a lowering of the energy, such that the free energy of the system decreases upon going from the metastable isotropic to the stable nematic phase, as required by thermodynamics. However, the Onsager model is a hard-core model: this means that, at a given temperature, the energy does not depend on density. 
Hence, all spontaneous phase transformations must involve an increase in entropy. In other words at a given density, the "ordered" nematic phase has a higher entropy than the "disordered" isotropic phase. The ordering of hard rods is an example of an entropic phase transition. Although counterintuitive at first, no laws of physics are violated: sure enough, the orientational entropy of the nematic phase is lower than that of the isotropic phase; however, this loss in orientational entropy is more than offset by the increase in translational entropy. As the particles align, their excluded volume decreases and hence they are able to explore a larger fraction of the volume without overlapping with other particles - this causes the translational entropy to increase.

A second important example of an entropic phase transition is the freezing transition of hard spheres. The first direct evidence for this transition came from the computer simulations of Wood and Jacobson, and Alder and Wainwright [5]. Initially, these results were received with a lot of scepticism (see, e.g. Ref. [6]); however, in recent years, it has become apparent that entropic phase transitions are quite common, not only in computer models, but also in real systems (mainly colloidal dispersions: see e.g. Ref. [7]). However, the concepts, the basic physics, were all contained in Onsager's paper.

\section{References}

1. Hansen JP, McDonald IR (1986) Theory of simple liquids, Academic Press, London

2. (a) Frenkel D (1987) J Phys Chem 91: 4912, (b) Frenkel D (1988) J Phys Chem 92: 5314

3. Khokhlov AR, Semenov AN (1981) Physica A 108: 546

4. Odijk T (1983) Macromolecules 16: 1340

5. (a) Wood WW, Jacobson JD (1957) J Chem Phys 27: 1207; (b) Alder BJ, Wainwright TE (1957) J Chem Phys 27: 1208

6. Percus JK (ed) (1963) The many-body problem. Interscience, New York

7. Pusey P (1991) In: Hansen JP, Levesque D, Zinn-Justin J (eds) Proceedings of the Les Houches summerschool session LI on: liquids, freezing and glass North-Holland, Amsterdam, pp 763 